\title{
Runtime Analysis and Instrumentation for Securing Software ${ }^{\star}$
}

\author{
R. Sekar \\ Department of Computer Science, Stony Brook University
}

\begin{abstract}
The past decade has witnessed an explosive increase in the scale, intensity and sophistication of cyber attacks. While software vendors have significantly increased their efforts on security, they are almost always playing "catch up." As a result, security-conscious organizations and individuals have come to expect their system administrators to deploy an array of tools and techniques to stay a step ahead of the hackers. While developer-oriented security tools rely mainly on static analysis, runtime analysis and policy enforcement are the mechanisms of choice in administrator-oriented tools. Runtime techniques offer increased automation and precision over static analysis, thus addressing the needs of administrators who don't have the time or resources needed to acquire extensive knowledge about the internals of a software system.

In this talk, I will begin by summarizing some of the significant advances that have been achieved in the past few years in the context of software vulnerability mitigation, including buffer overflow defenses, and more recently, the impressive results that have been achieved using dynamic information-flow analysis for blocking the most popular exploits today, including SQL and command injection and cross-site scripting. I will then proceed to describe dynamic analysis and enforcement techniques aimed at another high-profile security problem faced today, namely, malware defense. Our initial target in this regard has been on dynamic analysis techniques for extracting high-level models of program behavior. These models could be used in a variety of applications such as intrusion detection, vulnerability analysis and security policy verification. More recently, interesting advances have been made in the context of security policy development, where a combination of static and dynamic analysis techniques have been developed to synthesize low-level, enforceable policies that achieve a high-level goal such as protecting system integrity. Finally, I will conclude the talk with a discussion of some research opportunities and challenges in software security.
\end{abstract}

\footnotetext{
* This work was funded in part by ONR grants N000140110967 and N000140710928, and NSF grants CCF-0098154, CCR-0205376, CNS-0208877, CNS-0627687 and CNS-0831298.
} 